\title{
Electron Microscopic Study of Long-Term Denervated Rat Skeletal Muscle
}

\author{
DA-XING LU, ${ }^{1}$ SHI-KAI HUANG ${ }^{2}$ AND BRUCE M. CARLSON ${ }^{3 *}$ \\ 1Department of Anatomy and Cell Biology, University of Michigan, Ann Arbor, Michigan \\ 2I nstitute of Physiology, Shanghai, China \\ 3Institute of Gerontol ogy, University of Michigan, Ann Arbor, Michigan
}

ABSTRACT Background: This study describes the ultrastructure of long-term denervated rat extensor digitorum longus and tibialis anterior muscles, with particular emphasis on understanding the cellular basis for the reduced restorative capacity of Iong-term denervated muscle.

Methods: In $\mathbf{3 0}$ male WI/HicksCar rats, the right hindleg was denervated for periods of $1,2,4,5.5,6,7,12,14$, and 18 months before tissues were prepared for electron microscopy.

Results: Atrophy of muscle fibers was prominent by the second month post-denervation. At this time, type II fibers showed greater atrophy than type I fibers. At further periods of denervation, atrophy of all fibers was seen; and with increasing times of denervation the muscle fibers became surrounded by dense mats of collagen fibers. Muscle spindles persisted for the duration of this study. At two and four months, satellite cells showed signs of activation, such as elongated cytoplasmic processes and an increased concentration of cytoplasmic organelles. As denervation progressed, activated satellite cells became more widely separated from their associated muscle fibers, and basal lamina material was deposited between the satellite cells and muscle fibers. Some satellite cells broke free from their muscle fibers, and others acted as bridges between two muscle fibers. Evidence was seen of both muscle fiber degeneration and the regeneration of new muscle fibers, often more than one regenerating fiber beneath a single basal lamina. Loose folds of basal lamina were often present around atrophic muscle fibers. As denervation progressed, the morphology of individual muscle fibers varied. Some contained wellordered lattice arrays of myofilaments, whereas in others considerable sarcomeric disorganization was evident. Mitochondria became smaller and rounded; elements of the sarcoplasmic reticulum proliferated and became more disorganized; lipid droplets, glycogen deposits, and autophagic vesicles were all present in the cytoplasm of atrophic muscle fibers.

Conclusions: In addition to muscle fiber atrophy, long-term denervated muscles show evidence of myofiber and capillary death, as well as the deposition of massive amounts of interstitial collagen. These changes, all of which would appear to reduce the restorative capacity of the denervated muscle, take place concurrently with the morphological activation of satellite cells. The latter indicates that even in the denervated condition, restorative processes occur concurrently with regressive processes. Anat. Rec. 248:355-365, 1997.

Key words: denervation; skeletal muscle; rat; ultrastructure; atrophy

Following denervation, skeletal muscle undergoes a rapid loss in both mass and contractile force (Al-Amood and Lewis, 1989; Carlson et al., 1996; Gutmann, 1962). Denervation atrophy of muscle can be looked upon as an adaptive mechanism for reducing the load in a functionless region or as a response of the muscle fibers
Contract grant sponsor: NIH; contract grant number: PO1 AG10821.

*Correspondence to: Dr. Bruce M. Carlson, Department of Anatomy and Cell Biology, 4643 Medical Sciences II Bldg., University of Michigan, Ann Arbor, Michigan 48109.

Received 7 May 1996; accepted 12 February 1997. 
to a poor local environment. The restoration of a denervated muscle depends not only upon the return of a functional nerve supply, but also upon the ability of the muscle to respond by increasing its mass and contractile force. Both clinical experience (Harrison, 1989; Sunderland, 1978) and laboratory studies (Carlson et al., 1996; Gulati, 1990) have shown that after several months of denervation the restorative ability of skeletal muscle becomes sharply reduced. Attempts to improve the restorative ability of long-term denervated skeletal muscle (e.g., Billington, 1995a) depend upon a knowledge of the structural components and developmental capabilities of the muscle. Quantitative studies of satellite cells in denervated rat muscles (Rodrigues and Schmalbruch, 1995; Viguie et al., submitted) have shown an early increase in satellite cell number followed by a steady decline to considerably lower than control values by six to seven months of denervation; but other changes at both the cell and tissue level could contribute to the decline of I ong-term denervated muscle in terms of its restorative capacity.

The course of post-denervation events is complex. In addition to muscle fiber atrophy, dramatic changes occur in the vascular bed, leading to a severe topographic dissociation of the muscle fibers from their capillary supply, as well as sharply reduced capillary/ muscle fiber ratios (Borisov et al., 1996). Significant myonuclear loss and some myofiber death occur as well (Borisov and Carlson, 1995). A major accumulation of interstitial connective tissue foll owing denervation has the potential of influencing the exchange of materials between the vascular bed and muscle fibers, as well as affecting the extent of axonal ingrowth during reinnervation. This study was undertaken to identify the changes at the cell and tissue level that correspond to the al ready demonstrated reduction in the restorative capacity of long-term denervated muscles.

\section{MATERIALS AND METHODS}

This study was conducted on 30 male rats of the WI/HicksCar strain. At four months of age the right hindlimbs were denervated by severing the sciatic nerve high in the thigh. Before transection, the nerve was tightly ligated with 6-0 silk (Ethicon, Somerville, New J ersey) in two places separated by a half centimeter, and the nerve was cut between the sutures. Both proximal and distal ends of the nerve were deviated as far from one another as possible and implanted into muscular tissue. During surgery, the rats were anesthetized with ether. Postoperatively, the rats were treated for five days with terramycin that had been dissolved in their drinking water. This operative procedure produces consistent denervation for periods as long as 22 months (Carlson and Faulkner, 1988).

At intervals of $1,2,4,5.5,6,7,12,14$, and 18 months after denervation, groups of rats were euthanized and tissues were prepared for electron mi croscopic analysis. The denervated extensor digitorum longus (EDL) and tibialis anterior muscles were removed, pinned at resting length on a wax plate, and fixed by immersion in a solution containing $2.5 \%$ glutaral dehyde and $4 \%$ formaldehyde in $0.1 \mathrm{M}$ phosphate buffer at $\mathrm{pH}$ 7.4. One hour later, the muscles were cut into small blocks and fixed an additional four hours or overnight in fresh aldehyde fixative. The tissues were postfixed for one hour in $1 \%$ osmium tetroxide, dehydrated in a graded series of ethanol, and embedded in Spurr resin. Thin sections, cut in either transverse or longitudinal planes, were picked up on naked 90-mesh grids. After staining with uranyl acetate and lead citrate, the specimens were viewed with a Philips CM-10 electron microscope.

For histochemical analysis, muscles were frozen in liquid nitrogen and sectioned on a cryostat at $10 \mu \mathrm{m}$. The cross-sections were then reacted for alkaline $(\mathrm{pH}$ 10.4) and acid ( $\mathrm{pH} 4.3$ ) myosin ATPase activity according to the protocols of Guth and Samaha (1970) and Tunell and Hart (1977). F or immunocytochemical analysis, cryostat sections of frozen muscles were fixed in $2 \%$ paraformaldehyde and reacted with a mouse monoclonal antiibody to slow myosin $(\mathrm{A} 4.951$ [H. Blau] from the Developmental Studies Hybridoma Bank, Iowa City, I owa) and a rabbit polyclonal antibody to laminin (AB 949 from Chemicon, Tamecula, California). FITC-labelled secondary antibodies were, respectively, rat anti-mouse I gG (J ackson I mmunoresearch \#415-095-100) and goat anti-rabbit I gG (J ackson I mmunoresearch, West Grove, Pennsylvania \#111-095-144).

\section{RESULTS \\ Tissue Structure}

The tissue structure of the denervated EDL muscle corresponded to the pattern of loss in mass and contractile force reported elsewhere (Carlson et al., 1996). Atrophy of muscle fibers was prominent by the second month after denervation (Fig. 1B), but the degree of atrophy was not consistent among all muscle fibers. Both histochemical (myofibrillar ATPase) and immunocytochemical (antibody to slow myosin heavy chain) methods have demonstrated that type I (slow) muscle fibers undergo a slower course of atrophy than type II (fast) fibers (Fig. 2). The period between two and seven months of denervation was characterized by continued gross muscle and muscle fiber atrophy (Fig. 1C,D). By seven months of denervation, atrophy was already so severe that further changes in both muscle mass and muscle fiber diameter were much more gradual (Fig. 1E).

One of the most noticeable changes with increasing time of denervation was a steady increase in the amount of densely packed bundles of interstitial collagen fibers (Fig. 1). Interstitial collagen was present not only in perifascicular regions, but also in the endomysial spaces (e.g., Fig. 1E). With the increase in interstitial collagen, capillaries and other small blood vessels became surrounded by substantial layers of dense collagen, thereby isol ating them from the muscle fibers (Fig. 1D,E). The residue of degenerated capillaries was seen in long-term denervated muscles (Fig. 3). On a muscle-by-muscle basis, varying accumulations of fat cells and intrafiber lipid droplets were also seen after denervation (Fig. 1D).

Muscle spindles persisted throughout the period of denervation studied (Fig. 1D). As the post-denervation time progressed, morphological differences between nuclear bag and chain fibers became less, and the number of intrafusal fibers increased. Deposits of collagen fibers appeared both within the leaflets of the spindle capsule and among the intrafusal fibers, but these were not as dense as those that accumulated among the atrophying extrafusal fibers. 


\section{Satellite Cells and Their Relationships to Muscle Fibers}

By two months of denervation, individual satellite cells showed evidence of activation, such as long cytoplasmic processes extending between the muscle fiber surface and the surrounding basal lamina (Fig. 4). Elongated satellite cell processes were encountered throughout the denervation periods studied (Fig. 5). Occasionally, satellite cells in long-term denervated muscleextended cytoplasmic processes into the intersti tial space, as well (Fig. 6). By 14 and 18 months of denervation, the satellitecells possessed fewer cytoplasmic processes and assumed a morphological appearance more characteristic of the resting condition.

Relationships between satellite cells and their associated muscle fibers in long-term denervated muscle fell into several patterns beyond that typically seen in normal muscle. In one, the satellite cell remained in its original position, but the interspace between it and the muscle fiber was enlarged (Fig. 5). In a related pattern, the satellite cell was partially surrounded by cytoplasm of an intact muscle fiber, typically with an enlarged space between it and the muscle fiber. Satellite cells were also associated with degenerating muscle fibers, where both were enclosed within a common basal lamina. Varying degrees of interposition of basal Iamina material between satellite cells and muscle fibers were also seen. These ranged from the presence of partial-tocomplete interposed basal laminae (Fig. 7) to cases where satellite cells, completely enclosed in their own basal lamina, were connected to the parent muscle fiber by a partial common basal lamina, even though collagen fibers had formed between the satellite cell and the muscle fiber. Finally, satellite cells acted as bridges between two muscle fibers, with all components surrounded by a common basal Iamina (Fig. 8).

\section{Changes in Denervated Muscle Fibers}

By 18 months of denervation, muscle fiber diameters ranged from 2-15 $\mu \mathrm{m}$, in contrast to 30-80 $\mu \mathrm{m}$ in control fibers; and in addition to this substantial reduction in cross-sectional area, denervated muscle fibers showed a variety of characteristic changes. As the cross-sectional areas of the muscle fibers decreased with increasing denervation times, central nucleation became increasingly common. After nine months of denervation, many of the muscle fibers were central-nucleated. Starting at two months of denervation in the deep part of the EDL muscle and at four months in the superficial part, myotube-like fibers with central nuclei began to appear (Fig. 9). A common feature in two-month denervated muscle fibers was the appearance of hal os of filamentfree cytoplasm around certain nuclei (Fig. 10); the myonuclei themselves demonstrated crenation and folding of the nuclear membranes. In muscle fibers denervated between two and seven months, the condensation and fragmentation of nuclear chromatin was apparent in some nuclei (Fig. 11).

Within the cytoplasm of the muscle fibers, variation in the arrangement of the contractile filaments increased with increasing denervation time, and it was not uncommon for adjacent muscle fibers to have dramatic differences in sarcomere structure and overall appearance of the cytoplasm (Fig. 12). A well-ordered filament lattice was regularly seen in short-term dener- vated muscle (Fig. 13A). In long-term denervated muscles, fibers containing highly disorganized arrays of filaments were common (Fig. 13B); but even at 18 months of denervation, muscle fibers with well-ordered sarcomeres were seen (Fig. 12).

With increasing denervation time, mitochondria be camesmaller and more globular in shape, with sparsely arrayed cristae. The mitochondrial matrix often lost its normal staining density. The sarcoplasmic reticulum, likewise, underwent characteristic changes, becoming more irregular and losing its close relationship to the A-I junctions. Along with individual elements becoming expanded and vesiculated, the triads associated with the t-tubules became pentadic in structure. Lamellar inclusions (Fig. 6), reportedly derived from elements of the sarcoplasmic reticulum (Gori, 1972), were commonly seen in fibers from muscles denervated for over a year. Autophagic vesicles and lipid droplets were scattered among the cytopl asmic components of denervated muscle fibers, and deposits of glycogen granules were often prominent.

Relationships between muscle fibers and their basal laminae revealed several characteristic features. The presence of basal lamina material between satellite cells and the underlying muscle fiber has already been noted (Fig. 7). Folds of excess basal lamina were present around atrophic muscle fibers (Figs. 3 and 14), and it was not uncommon to see two or more muscle fiber cross-sections enclosed within a common basal lamina (Fig. 15). Often one of the muscle fibers exhibited a more immaturelooking ultrastructure than did its companion.

\section{DISCUSSION}

There are two main reasons for studying the mor phology of long-term denervated muscle. One reason is to determine if there is a morphological basis for the substantial decline in the restorative ability of skeletal muscle after several months of denervation. The second reason is to determine the nature of the muscle's response to denervation, in particular whether denervation atrophy consists of a steady decline and deterioration, or whether active cellular adaptative processes take place concurrently with the overall process of denervation atrophy.

In rats, quantitative studies on the restoration of long-term denervated muscle have mainly involved the transplantation of denervated muscles into normally innervated limbs of syngeneic hosts. Both morphological (Gulati, 1990) and physiological studies (Carlson et al., 1996) have shown a period of pronounced steady decline in the degree of restoration of grafts of EDL muscles that had been previously denervated for two to seven months. By seven months of denervation the level of restoration is so low that there is little room for further decline. Understanding the nature of events occurring during this period of dedine may help in the formulation of better methods for restoring denervated muscles.

Studies on the mass and gross contractile force of denervated rat muscles (Carlson et al., 1996; Gulati, 1990; Gutmann, 1962; Lewis and Schmalbruch, 1994) have shown that approximately $65 \%$ of the mass and $95 \%$ of the force is lost by two months, even though after transplantation a two-month denervated EDL muscle 

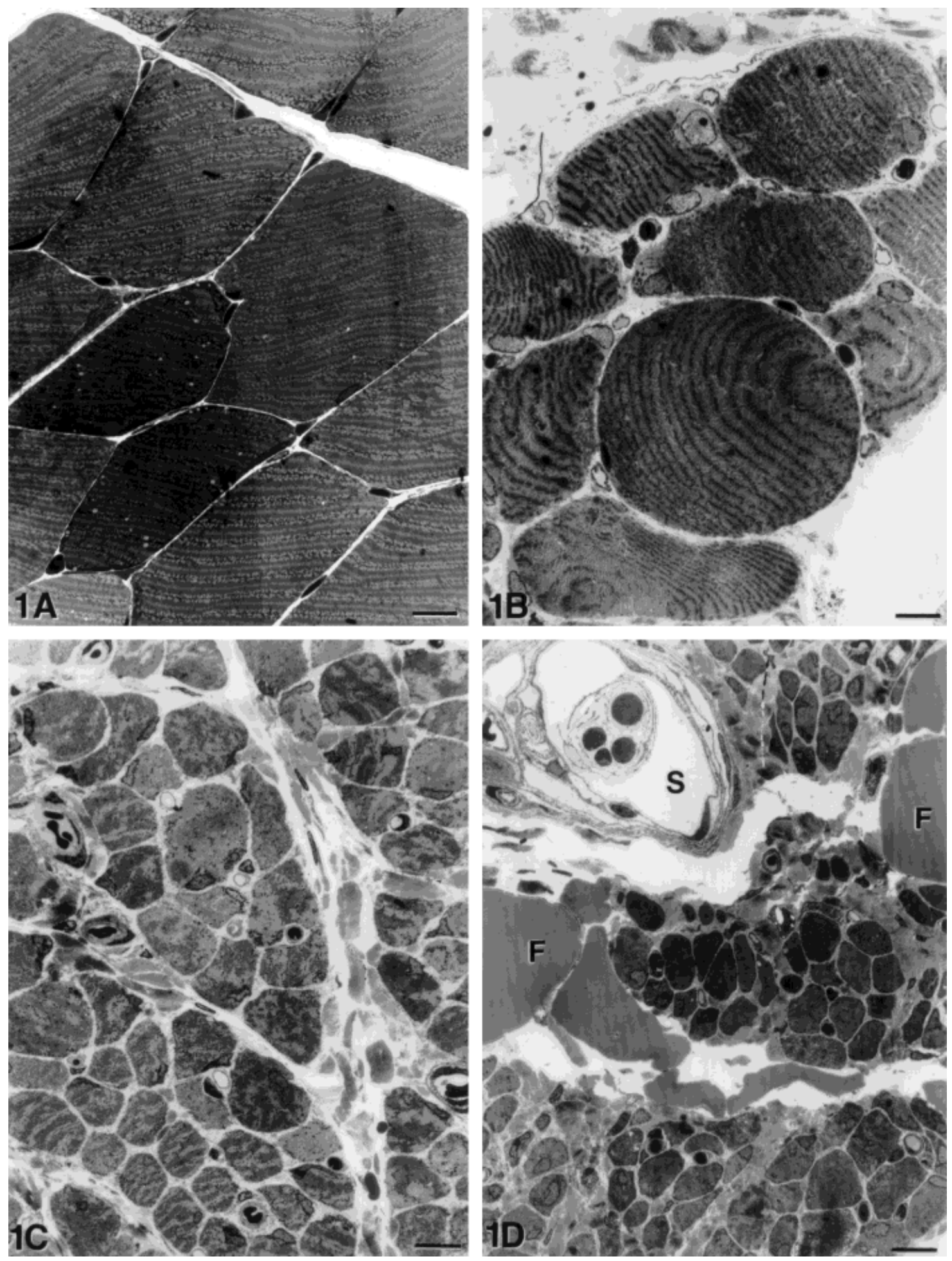

Fig. 1. Survey electron micrographs showing the progressive atrophy of denervated rat EDL muscle tissue. A: Control muscle from 4-month old rat. B: Two-month denervated muscle, showing differential atrophy of muscle fibers. The large round fiber is probably a type I fiber (See Fig. 2A). C: Four-month denervated muscle, showing an increase in endomysial and interfascicular connective tissue. D: Seven-month denervated muscle, showing continued muscle fiber atrophy, interstitial fat cells $(F)$, and a persisting muscle spindle (S).E: Eighteen-month denervated muscle, showing dense deposits of collagen fibers (asterisks and higher power insert) around severely atrophic muscle fibers. Bar $=10 \mu \mathrm{m}$. Bar on inset $=$ one um . 

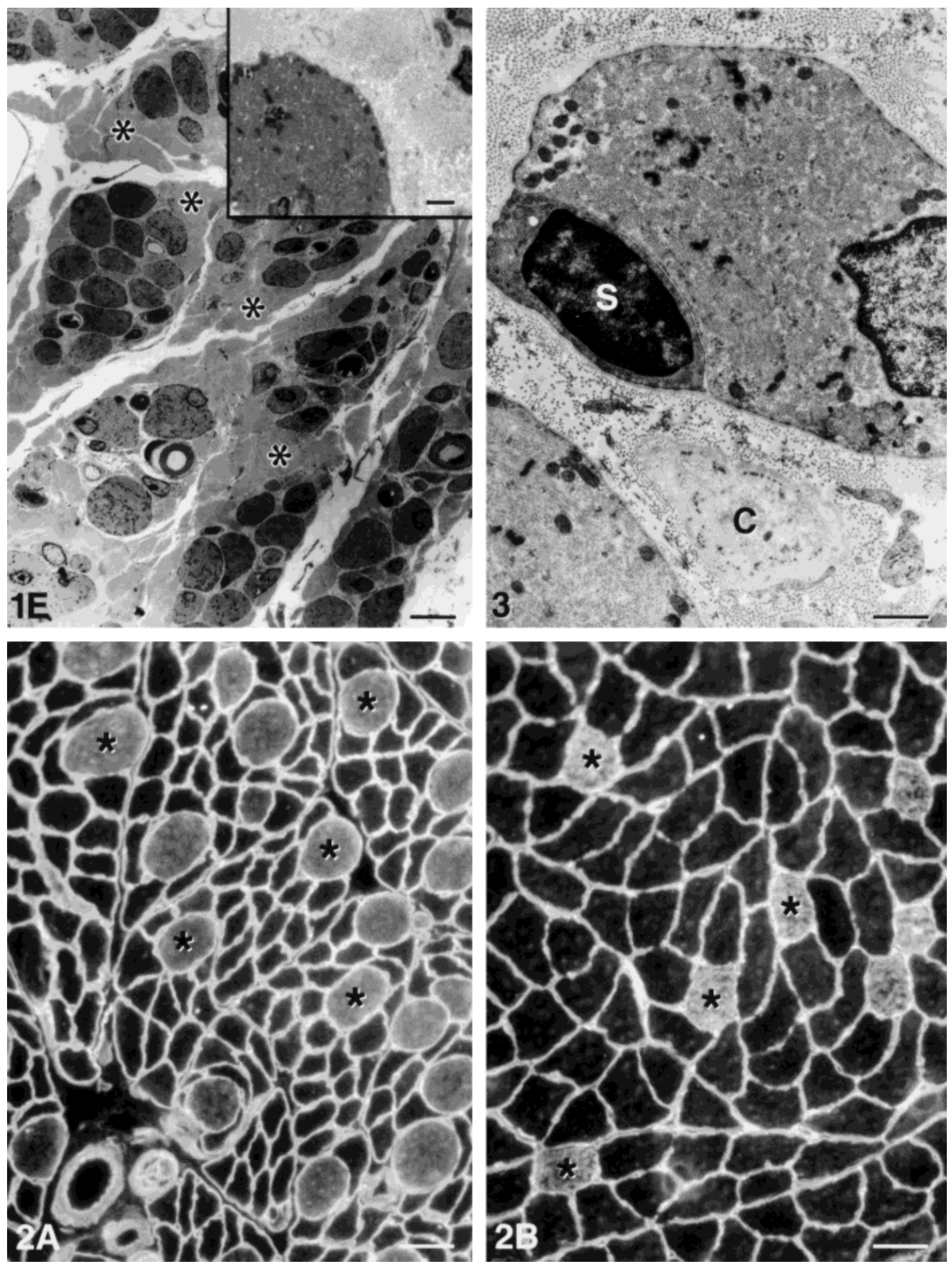

Fig. 1. Continued.

Fig. 2. A: Cross section of two-month denervated rat tibialis anterior muscle reacted with antibodies against laminin and slow myosin. Type I (slow) muscle fibers (asterisks) have become rounded, but have undergonelittle atrophy in relation to typell (fast) musclefibers, which are unstained but are bounded by Iaminin. B: Cross section of normal EDL muscle from a rat of the same age as the muscle depicted in A. Note the angularity of the type I muscle fibers (asterisks) and the much larger cross-sectional areas of the type II fibers. Bar = 30 um

Fig. 3. Electron micrograph of 18-month denervated rat EDL muscle, showing the basal lamina of a degenerated capillary (C) between two atrophic muscle fibers. Dense mats of collagen fibers surround the muscle fibers. S, satellite cell. Bar $=1 \mu \mathrm{m}$. 

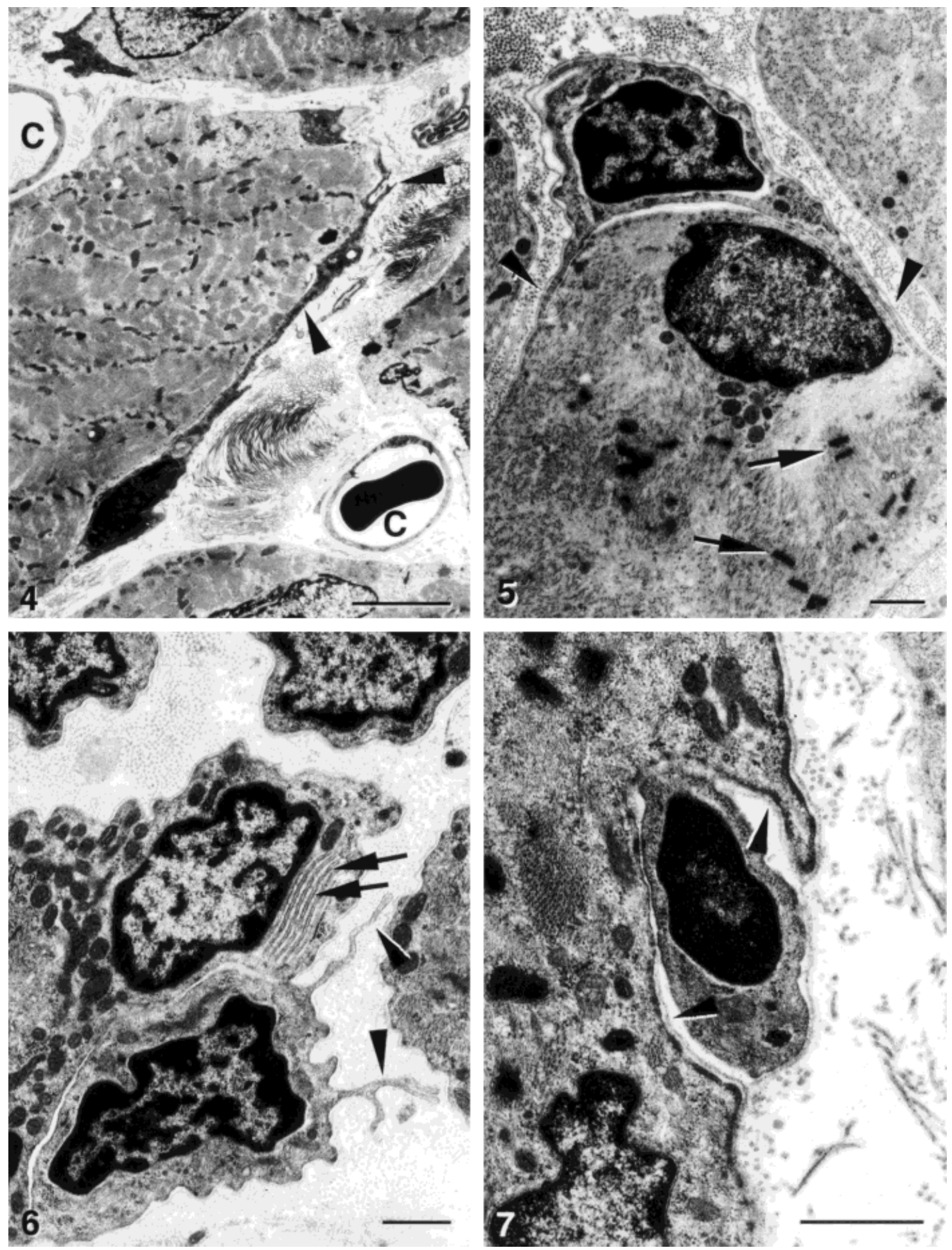

Fig. 4. Electron micrograph of two-month denervated rat EDL muscle, showing an activated satellite cell with a highly el ongated cytoplasmic process (arrowheads) extending over the surface of its associated muscle fiber. Capillaries (C) are still relatively normal in appearance, but collagen fibers are beginning to accumulate between muscle fibers. Bar $=5 \mu \mathrm{m}$.

Fig. 5. Electron micrograph of 12-month denervated rat EDL muscle, showing a satellite cell with two long cytoplasmic processes (arrowheads) extending over the surface of the muscle fiber. Note also the disorganization and misorientation of some of the sarcomeres (arrows) in the atrophic muscle fibers. Bar $=1 \mu \mathrm{m}$.

Fig. 6. Electron micrograph of seven-month denervated rat EDL muscle, showing a satellite cell extending long cytoplasmic processes (arrowheads) into the interstitial space. Within the associated muscle fiber, which is surrounded by dense deposits of collagen fibers, is a lamellar array (arrows). Bar = $1 \mu \mathrm{m}$.

Fig. 7. Satellite cell in 5.5-month denervated rat tibialis anterior muscle. A basal lamina (arrowheads) is interposed between the satellite cell and the muscle fiber, and the space between them is enlarged. Bar = $4 \mu \mathrm{m}$. 

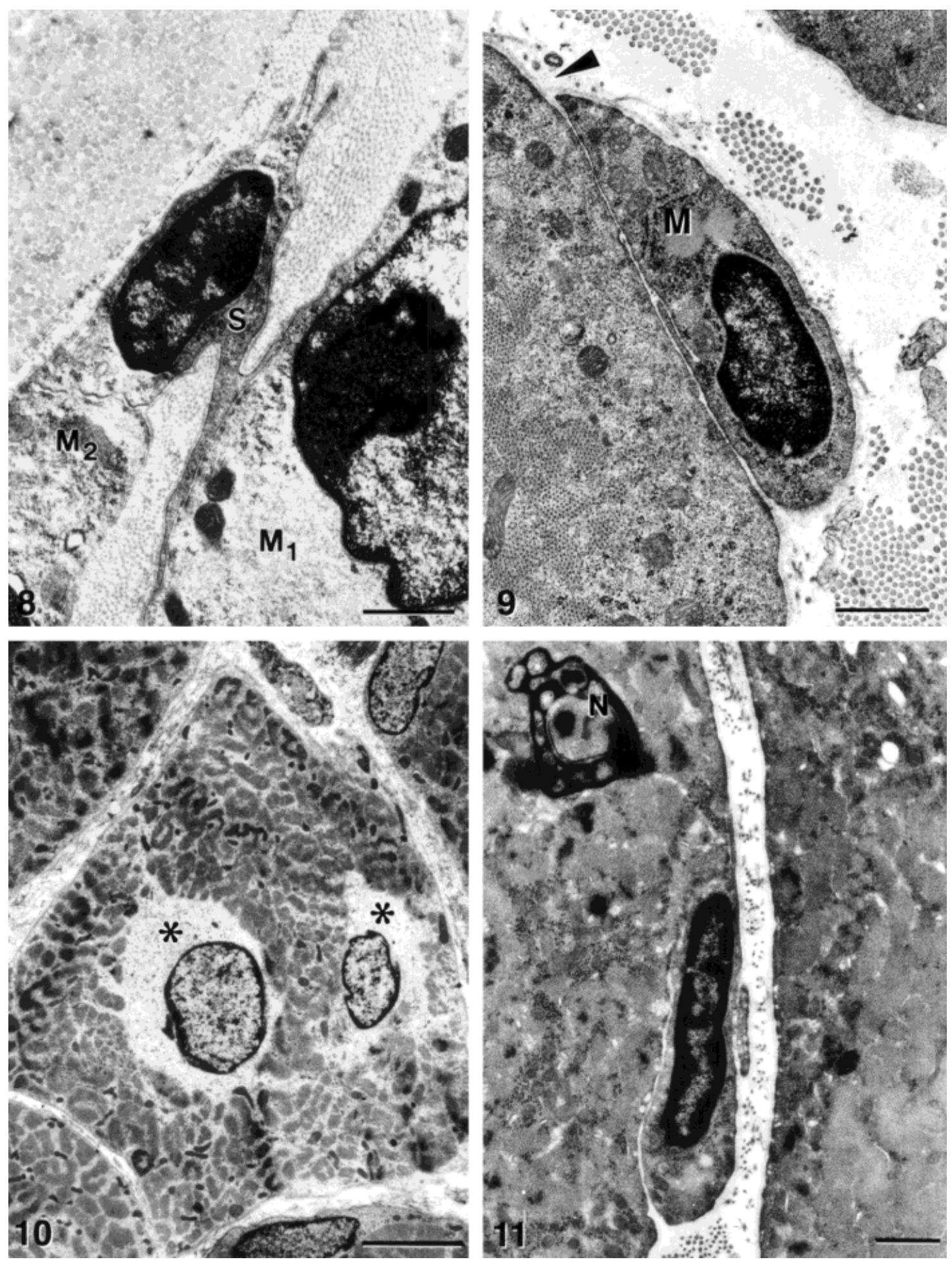

Fig. 8. Electron micrograph of a seven-month denervated rat EDL muscle showing an activated satellite cell (S) that is serving as a bridge between two other muscle fibers (M 1 and $M 2$ ) all beneath the same basal lamina. $\mathrm{Bar}=2 \mu \mathrm{m}$.

Fig. 9. Electron micrograph of 18-month denervated rat EDL muscle. An early myotube-like cell (M) is located next to a well-preserved muscle fiber. Both cells share a common basal lamina (arrowhead). Bar = $2 \mu \mathrm{m}$.

Fig. 10. Electron micrograph of two-month denervated rat EDL muscle. Halos of cytoplasm (asterisks) not containing myofilaments are present around two central myonuclei. Bar $=5 \mu \mathrm{m}$

Fig. 11. Electron micrograph of 18-month denervated rat EDL muscle. A centrally located myonucleus (N) shows a pattern of condensed chromatin characteristic of early nuclear degeneration. Bar $=2 \mu \mathrm{m}$. 

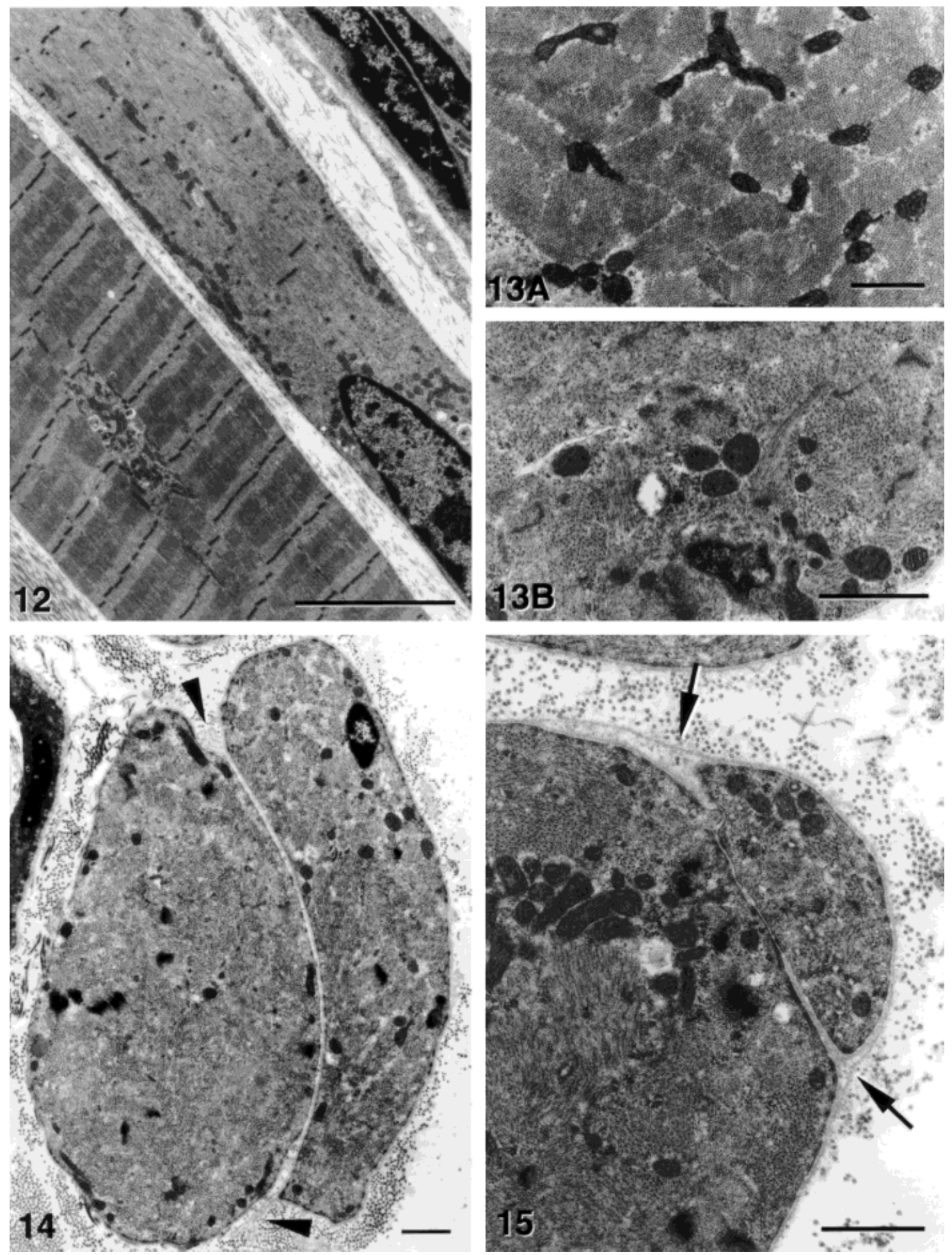

Fig. 12. Longitudinal section through muscle fibers in 18-month denervated rat EDL muscle. Note the differences in organization of sarcomeres between the two fibers. Bar $=5 \mu \mathrm{m}$.

Fig. 13. A: Cross section through the cytoplasm of a 2-month denervated rat EDL muscle fiber. Most of the myofilaments are still organized in a regular lattice array. Bar $=1 \mu \mathrm{m}$. B: Cross section through the cytoplasm of a 12-month denervated rat EDL muscle fiber. Some filaments run perpendicular to the long axis of the muscle fiber. The lattice organization of the cross-sectioned myofibrils is less regular than that of control or shorter-term denervated muscle. Bar $=1 \mu \mathrm{m}$.

Fig. 14. Cross section through two muscle fibers in an 18-month denervated rat EDL muscle. Folds of basal lamina are apparent (arrowheads). Bar $=1 \mu \mathrm{m}$.

Fig. 15. Two filament-containing muscle fibers within a common basal lamina in 18-month denervated rat EDL muscle. In some areas (arrows), duplication of the basal lamina is seen. Bar $=1 \mu \mathrm{m}$. 
becomes restored as well as a control muscle graft. Therefore, it is necessary to look for critical changes that occur in the muscle later than two months postdenervation.

Quantitative studies on numbers of myonuclei and satel lite cells have revealed an increase up to threefold over control values in both the percentage and absol ute number of satellite cells at the second month following denervation (Rodrigues and Schmal bruch, 1995; Viguie et al., submitted). This is followed by a steady ded ine to about one-third of the control number of satellite cells per muscle fiber by seven months after denervation (Viguie et al., submitted). Thereafter, the percentage of satellite cells (based upon the total number of myonuclei + satellite cells) stabilizes at slightly over $1 \%$ as long as 18 months after denervation. Rodrigues and Schmal bruch (1995) showed a relativel y greater decline in satellite cell percentage in the denervated rat soleus than in the EDL muscle.

Ontell (1975) has described four types of satellite cells in rat muscle denervated for up to three weeks. The first type is similar to those seen in normal muscle; the second type maintains the normal relationship between the muscle fiber and surrounding basal lamina, but the cell contains an increased amount of cytoplasm. These cells were seen as early as one week after denervation. The third and fourth types were only seen three weeks after denervation and consisted of cells with a greater or lesser degree of basal Iamina material between the satellite cell and the parent muscle fiber (type III) and degenerating satellite cells associated with degenerating muscle fibers (type IV). Cells of each of these types were seen in long-term denervated muscle, as well, suggesting that even 18 months after denervation the satellite cell population is functionally diverse.

The morphology of the satellite cells in denervated muscle suggests that many leave the quiescent stage and become activated. This is indicated by the elongated cytoplasmic processes associated with the satellite cells, as well as the increase in total amount of cytoplasm (Figs. 4 and 6). Mitotic figures in satellite cells were not seen in our study, but autoradiographic studies on denervated rodent muscle have shown increases in $\mathrm{H}^{3}$-thymidine incorporation into muscle fiberassociated nuclei. Murray and Robbins (1982 a,b) presented evidence of a peak of mitotic activity of all cell types in four-day denervated mouse EDL muscle. They noted the incorporation of $\mathrm{H}^{3}$-thymidine into satellite cells, along with the other cell types, but no specific numerical data on satellite cell labeling were presented. McGeachie and Allbrook (1978) also showed increased thymidine labeling of satellite cells during the week following denervation. There is very little information on mitotic activity of rat satel lite cells late in the first month and during the second month after denervation.

Corresponding with increased numbers and morphological activation of satellite cells is the appearance of some muscle fibers that have the morphology of early regenerating fibers. Satellite cells have been shown by Snow (1977) to beincorporated intoregenerating muscle fibers. Although quantitative studies were not done, it is our impression from parallel unpublished studies on soleus muscles from the same rats, as well as reports from the literature (Schmalbruch and Lewis, 1994), that after denervation the degeneration and regeneration of muscle fibers is more prominent in soleus than in EDL muscles.

The cause of muscle fiber degeneration after denervation remains obscure, but Borisov and colleagues (1996) have demonstrated a progressivereduction in the capillary/muscle fiber ratio in the months foll owing denervation that could account, at least in part, for the demise of existing muscle fibers. The presence of empty muscle fiber and capillary basal laminae in long-term denervated muscles is evidence for the degeneration without replacement of certain elements of denervated muscle. In other cases, however, it appears that single or even multiple rounds of muscle fiber degeneration and regeneration occur within a single muscle fiber complex, as suggested by the presence of multiple muscle fibers within a single or duplicated basal lamina tube (Fig. 15).

Muscle spindles were seen in muscles denervated as long as 18 months. The persistence of muscle spindles in rats for at least a year after denervation has al ready been reported (Gutmann and Zelená, 1962), and the morphology of the denervated spindles seen in this study corresponded with the data al ready summarized by Zelená (1994). Particularly prominent were the thickening of the capsule, an increase in the number of intrafusal fibers, and the reduction of differences between bag and chain fibers after long-term denervation. Although measurements were not made in this study, it was apparent that atrophy (reduction in cross-sectional area) of intrafusal fibers was, relatively, considerably less than that seen in extrafusal fibers, as has al ready been reported by Schröder and col leagues (1979).

The morphological evidence presented above shows that satel lite cells are active in denervated muscle, and that they would be potentially available for either the restoration of atrophic muscle fibers or for the regeneration of new muscle fibers. The findings from this and other studies (e.g., Schultz, 1978) suggests that, in denervated muscle, satellite cells can form new muscle fibers either within a pre-existing muscle basal lamina or by passing into the interstitium to form new independent muscle fibers. Quantitative data on satel lite cell populations (Viguie et al., submitted) suggest that the overall population of satellite cells in the EDL muscle declines after the second month of denervation; but whether or not the satellite cell population, by virtue of either its numbers or its proliferative potential, represents a limiting factor for restoration of long-term denervated muscle cannot be determined on the basis of presently available data.

Satellite cell numbers and the overall myogenic potential are important elements that determine the total restorative capacity of a long-term denervated muscle, but the overall myogenic potential cannot be realized unless motor nerve fibers are able to make functional contact with each muscle fiber. Studies on the restoration of denervated muscles through grafting them into innervated limbs have uniformly shown that grafts of muscles that had been denervated for less than threemonths contain homogeneous populations of large muscle fibers (Billington, 1995a,b; Carlson et al., 1996; Gulati, 1990). On the other hand, when the muscles had been denervated for periods of 4-12 months before 
grafting, the mature grafts have almost invariably consisted of fascicles of large, innervated muscle fibers alternating with fields of atrophic, presumably noninnervated muscle fibers. Such results strongly suggest the differential penetration of regenerating nerve fibers into the previously denervated muscles; these results also suggest that nerves do not grow into certain areas of the muscles. The finding of increasingly dense fields of collagen fibers around both muscle fibers and fascicles in denervated muscles (e.g., Fig. 1) suggests that the collagen fibers may act as a physical barrier to nerve ingrowth, and that the very small muscle fibers in grafts of previously denervated muscles were not contacted by nerves.

Another possibility for the poor restoration of longterm denervated muscles is that nerve fibers can grow to the muscle fibers, but are prevented by some characteristic of the basal laminae of the denervated muscle fibers from making functional contacts. Covault and colleagues (1987) have shown in vitro a preferential ability of denervated adult muscle, especially in the former endplate region, to support neurite outgrowth. This possibility cannot be addressed through a purely descriptive ultrastructural study such as the one reported here.

Still another option remaining to be tested is that in certain areas of long-term denervated muscle, the production of growth factors that facilitate either vascular or neural ingrowth is deficient, or that the surrounding connective tissue does not permit the diffusion of such factors. Several of these options, which could account for non-homogeneous degrees of restoration of different regions within denervated muscles, could be tested by sel ective removal of the connectivetissue that accumulates over months of denervation.

The accumulated data from this and other studies have all owed the formulation of a model that can serve as the basis for understanding and investigating the decline in the restorative capacity of a muscle foll owing denervation. The course of post-denervation atrophy can be divided into three phases: Phase 1, which includes the first two to three months after denervation, is a period in which the muscle undergoes profound atrophy, as measured by mass and maximum force. Nevertheless, during this period the denervated muscle is as capable of restoration as is control muscle, if transplanted into an innervated site (Carlson et al., 1996; Gulati, 1990). This high level of restorative capacity is correlated with a large increase in the number of satell ite cells (Viguie et al., submitted) and a high level of expression of myogenic regulatory molecules (Adams et al., 1995). During this period, a reduction in capillarity has begun (Borisov et al., 1996) but it is not yet profound, and large deposits of interstitial connective tissue have not yet been laid down. Phase $\mathbf{2}$ is the period from two to seven months after denervation, during which the restorative capacity of the muscle drops dramatically (Carlson et al., 1996). During this time there is a great loss of both myonuclei and satell ite cells (Viguie et al., submitted). Parallel to these changes are both a great loss in capillarity and a buildup of large deposits of densely packed collagen fibers in the interstitium. A principal characteristic of Phase 2 is the increasing predominance of secondary atrophic changes in the muscle. Phase $\mathbf{3}$ begins seven months after denervation and continues for the remainder of a prolonged denervation period. By this point, virtually everything measured (e.g., mass, contractile force, satellite cell numbers, capillary density, and the amount of interstitial connective tissue) has bottomed out to a stable basel ine state. Although some degree of reversal of the denervation atrophy can be accomplished by stimulating the muscle to regenerate(Billington and Carlson, 1996), recovery achieved to this point is far from complete.

\section{ACKNOWLEDGMENTS}

This study was supported by grant PO1 AG-10821 from the NIH. The authors thank Thomas Komorowski for technical assistance and Sandra Stewart for secretarial support.

\section{LITERATURE CITED}

Adams, L., B.M. Carlson, L. Henderson, and D. Goldman 1995 Adaptation of nicotinic acetylcholine receptor, myogenin, and MRF4 gene expression to long-term muscle denervation. J . Cell Biol., 5:1341-1349.

Al-Amood, W.S., and D.M. Lewis 1989 A comparison of the effects of denervation on the mechanical properties of rat and guinea-pig skeletal muscle. J. Physiol., 414:1-16.

Billington, L. 1995a The effect of regeneration on the functional restoration of denervated muscles. In: Proceedings of the 4th International Muscle Symposium. M. Frey and P. Giovanoli, eds. Universitätspital, Zürich, pp. 69-73.

Billington, L. 1995b Reinnervation and regeneration of denervated rat skeletal muscles. Ph.D. Thesis, University of Michigan, Ann Arbor.

Billington, L., and B.M. Carlson 1996 The recovery of denervated rat extensor digitorum longus muscles after Marcaine treatment and grafting. I. Neurol. Sci., 144:147-155.

Borisov, A.B., and B.M. Carlson 1995 Loss of nuclei in denervated skeletal muscle: Possible cellular mechanisms (abstr.). FASEB J ., 9:A825.

Borisov, A.B., S.-K. Huang, T.E. Komorowski, and B.M. Carlson 1996 Progressive microvascular insufficiency in long-term denervated skeletal muscle (abstr.). FASEB J ., 10:A533.

Carlson, B.M., L. Billington, and J. A. Faulkner 1996 Studies on the regenerative recovery of long-term denervated muscle in rats. Restor. Neurol. Neurosci, 10:77-84.

Carlson, B.M., and J.A. Faulkner 1988 Reinnervation of long-term denervated rat muscle freely grafted into an innervated limb. Exp. Neurol., 102:50-56.

Covault, J., J.M. Cunningham, and J.R. Sanes 1987 Neurite outgrowth on cryostat sections of innervated and denervated skeletal muscle. J . Cell Biol., 105:2479-2488.

Gori, Z. 1972 Proliferations of the sarcoplasmic reticulum and the T system in denervated muscle fibers. Virchows Arch. Abt. B Zellpath., 11:147-160.

Gulati, A.K. 1990 Restoration of denervated skeletal muscle transplants after reinnervation in rats. Restor. Neurol. \& Neurosci., 2:23-29.

Guth, L., and F.J. Samaha 1970 Procedure for the histochemical demonstration of actomyosin ATPase. Exp. Neurol., 28:365-367.

Gutmann, E. (ed.) 1962 The Denervated Muscle. Praha, Publishing House of Czechoslovak Acad. Sci., Prague, Czechosl ovakia.

Gutmann, E., and Zelená, J. 1962 Morphological changes in the denervated muscle. In: The Denervated Muscle. E. Gutmann, ed. Academia, Prague, pp. 57-102.

Harrison, D. 1989 Current trends in the treatment of established unilateral facial palsy. F unctional Surgery of the Head and Neck. E. Karcher, ed. Graz, pp. 9-16.

Lewis, D.M., and H. Schmalbruch 1994 Contractile properties of aneurally regenerated compared with denervated muscles of rat. J . Muscle Res. and Cell Motil., 15:267-277.

McGeachie, J., and D. Allbrook 1978 Cell proliferation in skeletal muscle following denervation or tenotomy. Cell Tissue Res., 193:259-267.

Murray, M.A., and N. Robbins 1982a Cell proliferation in denervated muscle: Time course, distribution and relation to disuse. Neuroscience, 7:1817-1822. 
Murray, M.A., and N. Robbins 1982b Cell proliferation in enervated muscle: I dentity and origin of dividing cells. Neuroscience, 7:18231833.

Ontell, M. 1975 Evidence for myoblastic potential of satellite cells in denervated muscle. Cell Tissue Res., 160:345-353.

Rodrigues, A. de C., and H. Schmalbruch 1995 Satellite cells and myonuclei in long-term denervated rat muscles. Anat. Rec., 243:430-437.

Schmalbruch, H., and D.M. Lewis 1994 A comparison of the morphology of denervated with aneurally regenerated soleus muscle of rat. J. Muscle Res. and Cell Motil., 15:256-266.

Schröder, J .M., P.T. Kemme, and L. Scholz 1979 The fine structure of denervated and reinnervated muscle spindles: Morphometric study of intrafusal muscle fibers. Acta Neuropathol. (Berl.), 46:95-106.
Schultz, E. 1978 Changes in the satellite cells of growing muscle following denervation. Anat. Rec., 190:299-312.

Snow, M.H. 1977 Myogenic cell formation in regenerating rat skeletal muscle injured by mincing. II. An autoradiographic study. Anat. Rec., 188:201-218.

Sunderland. S. 1978 Nerve and Nerve Injuries, 2nd Ed. ChurchillLivingston, Edinburgh.

Tunell, G.L., and M.N. Hart 1977 Simultaneous determination of skeletal muscle fiber types I, II A and II B by histochemistry. Arch. Neurol., 34:171-173.

Viguie, C.A., D.-X. Lu, S.-K. Huang, and B.M. Carlson A quantitative study of the effects of long-term denervation on the extensor digitorum longus muscle of the rat. Anat. Rec., submitted.

Zeliná, J . 1994 Nerves and Mechanoreceptors. Chapman \& Hall (eds.), London. 